\title{
Selection of multiresistant coliforms by long-term treatment of hypercholesterolaemia with neomycin
}

\author{
M V VALTONEN, R J SUOMALAINEN, R H YLIKAHRI, V V VALTONEN
}

British Medical fournal, 1977, 1, 683-684

\section{Summary}

Patients with hypercholesterolaemia are often treated with the antimicrobial agent neomycin. Such treatment is potentially dangerous, however, as it may favour the emergence of multiresistant, $R$-factor-carrying, enteric bacteria among the intestinal flora. In 11 out of 14 patients who had received neomycin for three months to eight years most of the faecal coliforms were resistant to at least four antimicrobial drugs and capable of transferring this resistance to others. In contrast, only one out of nine patients who were treated with other lipid-lowering drugs had resistant bacteria in their faeces. Neomycin may cause multiresistant strains to emerge because, like tetracycline, it forms high concentrations in the gut. Long-term treatment of non-infectious conditions like hypercholesterolaemia with neomycin is potentially dangerous not only to the patient but also to the community because of the creation of a reservoir of multiresistant organisms.

\section{Introduction}

While the number of bacteraemic infections caused by enteric bacteria has increased, especially among patients with a serious underlying disease, ${ }^{1}$ these bacteria have become increasingly resistant to various antimicrobial agents. ${ }^{2}{ }^{3}$ In most cases this resistance is caused by transferable resistance $(R)$ factors. ${ }^{4-6}$ As oral treatment with antimicrobial agents seems to be the main cause of the spread of $\mathrm{R}$-factors, ${ }^{7-9}$ the only way of stopping or reversing this spread is to restrict the use of antimicrobial agents.

We have been concerned about the considerable "hidden" use of antimicrobial agents for treating non-infectious diseases. For example, long-term treatment of acne vulgaris with antimicrobial agents led to the emergence of $\mathrm{R}$-factor-carrying intestinal flora that were resistant to several drugs in almost every patient. ${ }^{10}$ We therefore investigated patients with hypercholesterolaemia to see whether long-term neomycin treatment had a similar effect.

\section{Patients and methods}

Each consecutive outpatient who was treated at the University Central Hospital of Helsinki for hypercholesterolaemia (mean initial serum cholesterol $12.7 \mathrm{mmol} / 1$ (490 mg/100 ml); range $10 \cdot 4-14.6$ $\mathrm{mmol} / \mathrm{l}(402-564 \mathrm{mg} / 100 \mathrm{ml}))$ and who had received no antimicrobial

Central Public Health Laboratory, Helsinki, Finland

$M$ V VALTONEN, $M D$, research and teaching assistant

R J SUOMALAINEN, MD, research and teaching assistant

Second and Third Department of Medicine, University Central Hospital, Helsinki, Finland

R H YLIKAHRI, MD, senior lecturer in medical chemistry $\mathrm{V} V$ VALTONEN, MD, senior lecturer in internal medicine treatment other than neomycin for at least a year was included in the study. We divided these patients into two groups according to whether they had been treated with neomycin. Nine men aged 31-55 years who had been treated for at least three months with non-antimicrobial lipid-lowering drugs (clofibrate or nicotinic acid) formed a control group. They continued their treatment during the study. The remaining 14 patients (six women, eight men aged 27-64 years) had been treated for at least three months with neomycin $(500 \mathrm{mg}$ orally three times a day). They also continued their treatment throughout the study.

One sample of faeces was collected from each patient. Each sample was plated in tenfold dilutions on nutrient agar (Oxoid) plates and on two kinds of selective nutrient agar plates, ${ }^{10}$ containing either tetracycline $(10 \mathrm{mg} / \mathrm{l})$ or neomycin $(10 \mathrm{mg} / \mathrm{l})$. The numbers of colonies with coliform morphology were estimated on each plate. Two colonies were picked at random from each of the selective plates and restreaked on nutrient agar for single colony isolation. If these proved to be Escherichia coli (standard biochemical tests) they were tested for sensitivity to sulphonamides, tetracyclines streptomycin, neomycin, ampicillin, gentamicin, and carbenicillin, ${ }^{11}$ using standard discs (Biodisk).

All different isolates from each sample were then tested for transfer of antimicrobial resistance to $E$ coli in a mixed culture, as described elsewhere. ${ }^{10}$ Briefly, the recipient $E$ coli was sensitive to the abovementioned drugs but resistant to sodium azide, to which all the patient isolates were sensitive. Transfer of resistance was detected on nutrient agar plates containing azide and the appropriate drug $(10 \mathrm{mg} / \mathrm{l})$ and confirmed by testing colonies isolated from these plates.

\section{Results}

No resistant bacteria were found in eight of the nine controls (fig 1), while $30^{\circ}{ }_{0}^{\circ}$ of the aerobic flora in one sample (case 3 ) consisted of coliforms resistant either to sulphonamides, tetracyclines, and ampicillin or to tetracyclines, streptomycin, neomycin, and ampicillin. ${ }^{8}$ In the first type the resistance was transferred to sulphonamides, tetracycline, and ampicillin, and in the second group it was transferred to ampicillin.

The predominant aerobic flora in all 14 patients who were treated with neomycin was resistant to neomycin (fig 2). The resistant bacteria were coliforms in 11 patients and enterococci or staphylococci in the

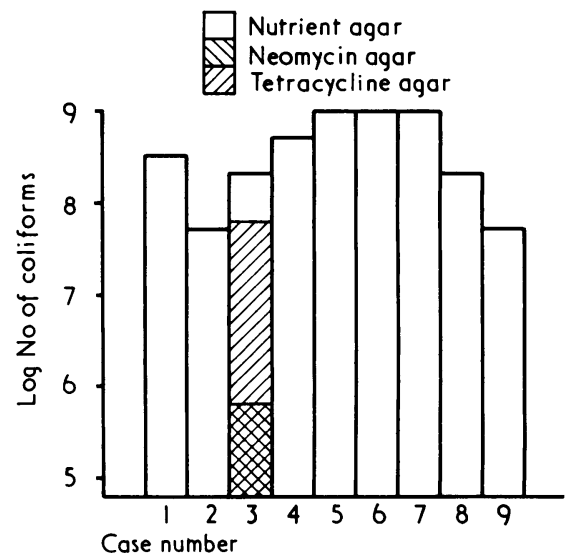

FIG 1-Log number of coliform colonies in faecal samples from controls that grew on nutrient agar and on selective media containing either neomycin $(10 \mathrm{mg} / \mathrm{l})$ or tetracycline (10 $\mathrm{mg} / 1)$. 


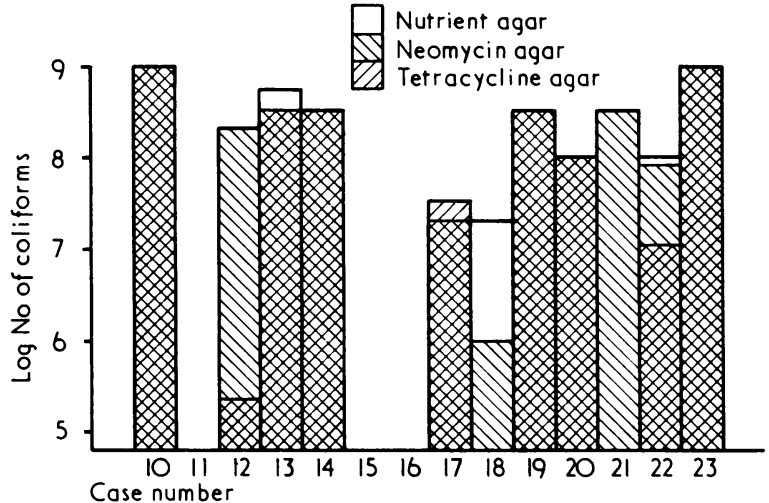

FIG 2-Log number of coliform colonies in faecal samples of patients treated with neomycin that grew on nutrient agar and on selective medium containing either neomycin $(10 \mathrm{mg} / \mathrm{l})$ or tetracycline $(10 \mathrm{mg} / \mathrm{l})$. Multiresistant staphylococci and enterococci were predominant organisms in samples from cases 11,15 , and 16.

Resistance pattern of predominant coliform strain in samples from patient receiving neomycin

\begin{tabular}{|c|c|c|}
\hline Case No & Predominant ${ }^{1}$ strain resistant to: & Transfer of resistance \\
\hline $\begin{array}{l}10 \\
11 \\
12 \\
13 \\
14 \\
15 \\
16 \\
17 \\
18 \\
19 \\
20 \\
21 \\
22 \\
23\end{array}$ & $\begin{array}{l}\text { Su, T, S, N, A, C } \\
\text { * } \\
\text { S, N, A, C } \\
\text { Su, T, N, A, C } \\
\text { T, S, N, A, C } \\
\text { * } \\
\text { Su, T, S, A, C } \\
\text { Su, S, N, A, C } \\
\text { Su, T, S, N, A, C } \\
\text { Su, T, S, N, } \\
\text { Su, S, N, A, C } \\
\text { Su, T, S, N, A, C } \\
\text { Su, T, S, N, A, C }\end{array}$ & $\begin{array}{l}\text { Su, T, S, N, A, C } \\
\text { Nt } \\
\text { S, N, A, C } \\
\text { T, N } \\
\text { T, S, N, A, C } \\
\text { Nt } \\
\text { NT } \\
\text { Su, A C } \\
\text { Su, T, S, A, C } \\
\text { Su, T, S, N, A, C } \\
\text { Su, T } \\
\text { S } \\
\text { S, A, C }\end{array}$ \\
\hline
\end{tabular}

${ }^{1}$ As judged on the difference of viable counts on selective and nonselective plates. The sensitivity pattern was that of strains picked from selective plates containing The sensitivin.

Su=Sulphonamides. $\quad \mathrm{T}=$ Tetracyclines. $\mathrm{S}=$ Streptomycin. $\mathrm{N}=$ Neomycin. $\mathrm{A}=$ Ampicillin. $\mathrm{G}=$ Gentamicin, $\mathrm{C}=$ Carbenicillin. NT $=$ Not tested.

*Multiresistant staphylococci and enterococci.

remaining three. Most of the coliforms were resistant to at least four of the drugs tested and also transferred most of the resistance determinants (see table). All the resistant bacteria were, however, still sensitive to another aminoglycoside, gentamicin.

\section{Discussion}

Neomycin has been widely used to treat familial hypercholesterolaemia. It is practically non-absorbable and therefore exerts its effects in the gut. Its effect on the plasma cholesterol level is thought to be due to its polybasic chemical structure rather than to its antimicrobial properties. It precipitates ionised fatty acids and bile acids, thus disrupting the micellar phase of the intestinal contents, decreasing the absorption of cholesterol, ${ }^{12}{ }^{13}$ and increasing cholesterol excretion in faeces. ${ }^{\mathbf{1 4}}$

We have shown that long-term treatment with neomycin has a profound effect on the aerobic intestinal flora. In $80^{\circ}{ }^{\circ}$ of the patients receiving neomycin almost all aerobic bacteria in the faeces were resistant to four or more antimicrobial agents. All the multiresistant strains were also capable of transferring their resistance to $E$ coli. By comparison, only one of the nine patients who were not receiving neomycin had resistant bacteria.

Tetracycline is another drug that is particularly effective in selecting resistant $\mathrm{R}$-factor-carrying strains. ${ }^{9}$ Even small doses are enough to select such multiresistant strains. ${ }^{10}$ Both tetracycline and neomycin form high concentrations in the gut, tetracycline because of the enterohepatic circulation ${ }^{15}$ and neomycin because it is not absorbed. It may be these high concentrations that make both drugs effective in producing resistant organisms.

Long-term treatment for hypercholesterolaemia with neomycin increases the potential danger of resistant flora both to the patients themselves and, more generally, to the community. The patients are at risk of, for example, ascending urinary tract infections and gram-negative bacteraemia, which may be hard to treat because of the resistance of the strains. And the community is at risk because a reservoir of $R$ factors has been created.

We wish to thank Hannele Kiviaho and Marianne Hovi for technical help.

\section{References}

${ }^{1}$ Finland, M, fournal of Infectious Diseases, 1970, 122, 419.

2 Datta, N, British Medical fournal, 1969, 2, 407.

${ }^{3}$ Simmons, H E, and Stolley, P D, Fournal of the American Medical Associaiion, 1974, 277, 1023.

4 Anderson, E S, Annual Review of Microbiology, 1968, 22, 131.

${ }^{5}$ Jonsson, M, Scandinavian fournal of Infectious Diseases, 1972, 4, 133.

6 Watanabe, T, Bacteriological Review, 1963, 27, 87.

' Hartley, C L, and Richmond, M H, British Medical fournal, 1975, 4, 71.

8 Jonsson, M, Scandinavian fournal of Infectious Diseases, 1974, 6, 339.

${ }^{9}$ Datta, N, et al, Lancet, 1971, 1, 312.

10 Valtonen, M V, et al, British fournal of Dermatology, 1976, 95, 311.

${ }^{11}$ Ericson, H, Högman, C, and Wichman, K, Scandinavian fournal of Clinical and Laboratory Investigation, 1954, 6, Suppl No 11, p 21.

${ }^{12}$ Miettinen, T A, in Proceedings of the Sixth International Congress of Pharmacology, ed J Tuomisto and M K Paasonen, vol 4, p 149. Oxford, Pergamon Press, 1976.

13 Thompson, R R, Macmahon, M, and Claes, P, European fournal of Clinical Investigation, 1970, 4, 40.

${ }^{14}$ Miettinen, T A, in Lipid Metabolism and Atherosclerosis, International Congress Series No 238. Amsterdam, Excerpta Medica, 1973.

15 Garrod, L, Lambert, H, and O'Grady, F, (editors), Antibiotics and Chemotherapy, 4th edn, p 149. Edinburgh and London, Churchill Livingstone, 1973.

(Accepted 13 fanuary 1977) 